\title{
Le monde rural : un concept en évolution
}

Jean-Claude Bontron

\section{OpenEdition}

Journals

Édition électronique

URL : http://journals.openedition.org/ries/3303

DOI : 10.4000/ries.3303

ISSN : 2261-4265

\section{Éditeur}

Centre international d'études pédagogiques

\section{Édition imprimée}

Date de publication : 1 juin 1996

Pagination : 25-30

ISSN : 1254-4590

\section{Référence électronique}

Jean-Claude Bontron, "Le monde rural : un concept en évolution », Revue internationale d'éducation de Sèvres [En ligne], 10 | 1996, mis en ligne le 30 juillet 2013, consulté le 19 avril 2019. URL : http:// journals.openedition.org/ries/3303 ; DOI : 10.4000/ries.3303

Ce document a été généré automatiquement le 19 avril 2019

(c) Tous droits réservés 


\title{
Le monde rural : un concept en évolution
}

\author{
Jean-Claude Bontron
}

1 Le monde rural peut sembler à certains ancré dans les valeurs du passé et voué à un déclin irréversible face à la montée de l'urbanisation. C'est en réalité en avoir une vision très superficielle car il est entré dans une phase de profonde transformation qui le place dans le champ du développement économique général, des modes de vie et des aspirations de la société contemporaine.

\section{Universalité du fait rural}

2 L'universalité du fait rural n'est plus à démontrer. Le terme est utilisé depuis fort longtemps dans pratiquement tous les pays du monde pour désigner les organisations sociales agraires ou les régions d'habitat dispersé. Le concept a été et demeure en partie le fondement d'une division majeure dans les sciences sociales comme l'histoire, la géographie, la sociologie ou l'économie ; le rural est une catégorie des représentations sociales très largement présente dans le débat politique et dans l'action publique d'aménagement du territoire.

3 Cette notion s'appuie sur trois dimensions fondatrices: la prééminence des activités agricoles et pastorales dans les formes de l'organisation économique et sociale; une spécificité du système de valeurs (rôle de la famille, de la propriété du sol, de la tradition...) et des modes de vie (autoconsommation, travail, solidarités villageoises...) par rapport aux habitants des villes ; les particularités d'un mode de colonisation de l'espace et d'utilisation des ressources du sol qui aboutit à une occupation extensive du territoire avec pour corollaire la dispersion et la faible densité du peuplement.

4 Ces trois éléments, qui jouent un rôle plus ou moins important selon le niveau de développement des pays, sont en partie remis en cause par les évolutions actuelles de la société dans beaucoup d'entre eux. Les transformations de l'organisation économique, des modes de vie, des pratiques résidentielles changent en effet profondément le rapport des hommes à leur territoire, et les différences communément admises entre villes et 
campagnes en viennent à être contestées. Ainsi, l'agriculture ne peut plus être le fondement de la ruralité car les espaces ruraux sont aujourd'hui très minoritairement peuplés d'agriculteurs et parce que la vocation agricole de ces espaces est parfois même reléguée au second plan par la montée d'une demande sociale pour d'autres usages (loisirs, conservation de la nature...). Le mode de vie rural, s'il a jamais existé en tant que tel, se dissout aujourd'hui dans un modèle de consommation généralisé, dont les valeurs et les aspirations sont partagées selon des clivages qui n'ont guère à voir avec des différences villes/campagnes mais plutôt avec les niveaux de revenus. Par contre, la diversification en cours des fonctions de l'espace rural n'a que peu modifié les modalités de son occupation, et la faible densité comme la faible valeur foncière demeurent des dimensions fortes de la distinction rural/urbain.

5 Si la notion de rural est communément admise et utilisée, il n'existe pas partout une définition officielle pour désigner les territoires concernés. Les définitions utilisées dans chaque pays présentent des différences sur de nombreux points et ne permettent pas une véritable approche comparative. Le fait qu'elles soient souvent le négatif de la définition des villes est d'ailleurs un symptôme de la difficulté à cerner les attributs qui soient propres au milieu rural. C'est la raison pour laquelle l'OCDE a entrepris dans le cadre de son "programme de développement rural » un travail conceptuel et pratique, visant à établir une définition harmonisée des "communautés rurales » sur la base de la densité de population et à promouvoir une typologie des régions en fonction de leur degré de ruralité1. La réalisation de ce système par un groupe d'experts a permis de proposer tout un jeu d'indicateurs à partir duquel il est maintenant possible d'établir des comparaisons entre les régions rurales des pays membres. C'est sur cette base que nous évoquerons les quelques données chiffrées qui vont suivre.

6 Il ressort de l'analyse que l'on peut conduire à partir des indicateurs de l'OCDE que le fait rural est partout présent, avec cependant un poids économique et social fort différent selon les pays. Si l'on considère les régions qualifiées dans la typologie d'« essentiellement rurales », le gradient est extrêmement ouvert: entre $15 \%$ du territoire national (Belgique) et $91 \%$ (Irlande) pour l'Europe; $92 \%$ et $95 \%$ pour l'Australie et le Canada qui possèdent de vastes espaces à très faible peuplement. Leur poids démographique est naturellement moins important, mais oscille entre $2 \%$ (Belgique) et $62 \%$ (Irlande).

7 Il faut en rechercher les différences dans le rôle de la nature (présence de vastes espaces montagneux ou désertiques), mais plus encore dans celui de l'histoire des peuples qui ont colonisé et mis en valeur leur espace selon des modalités très variées et concrétisé leur volonté de socialisation dans des formes d'urbanisme qui vont de simples réseaux de petites villes à de vastes conurbations métropolitaines. Cette urbanisation croissante n'annonce pas la fin de la société rurale aujourd'hui redécouverte et réhabilitée par de forts courants migratoires et par un renversement de sa place dans une échelle de valeurs qui privilégie la nature, le patrimoine, le cadre de vie et voit dans la ruralité renouvelée une alternative à la crise des sociétés urbaines².

\section{Extrême diversité des situations}

8 La notion d'espace rural n'a cependant de pertinence que si l'on admet la grande diversité des situations qu'il recouvre, et notamment le fait qu'il n'est pas nécessairement lié à l'agriculture. 
9 Le premier aspect de cette diversité rurale a trait aux formes concrètes de son peuplement et à l'armature des bourgs et villes qui l'animent. Dans nos pays de vieille tradition agraire de l'Europe moyenne, où une large partie du territoire a été mise en valeur selon des systèmes de polyculture et d'élevage, où de petites industries ont pris le relais de l'exode paysan, les densités démographiques des zones rurales varient autour de quarante à cinquante habitants au kilomètre carré. Mais si l'on pense à certaines vallées de haute montagne, aux landes d'Écosse, aux plateaux castillans où l'élevage extensif et la céréaliculture sèche sont les seules ressources, la densité oscille autour de dix habitants au kilomètre carré ; elle descend à un ou deux dans les espaces ruraux des clairières forestières de l'Europe du Nord. À l'inverse, dans les régions « jardinées » de certains pays du Sud, cette densité peut dépasser cent habitants au kilomètre carré. Malgré ce large gradient, nous sommes toujours très loin des densités urbaines qui se comptent plutôt en milliers d'habitants au kilomètre carré.

10 À des niveaux de densité voisins, l'habitat peut également présenter des formes très variées d'implantation allant d'une extrême dispersion des maisons (comme en Irlande ou dans les bocages français de l'Ouest) à une structure de quelques milliers d'habitants (comme les bourgs alsaciens ou les pueblos anda-lous), et parfois jusqu'à de véritables villes rurales régnant sur un désert agricole (Italie du Sud). Autant de conditions qui, on peut l'imaginer, vont fortement conditionner l'organisation de l'école.

11 Le second aspect concerne la structure même de l'économie. Si, dans les pays les moins développés, l'activité rurale gravite presque exclusivement autour de l'agriculture, ce n'est plus le cas dans la majorité des autres pays. L'économie s'est largement diversifiée par l'installation d'industries agroalimentaires ou d'industries qui sont fortes utilisatrices de main-d'œuvre (bois, textile, sous-traitances diverses), par le tourisme qui, là où il s'est développé significativement, est créateur d'emplois directs et indirects. Par ailleurs, l'économie des services a tardivement mais largement pénétré le milieu rural, autour notamment du secteur de la santé.

12 Au total, l'agriculture apparaît actuellement très minoritaire par l'offre d'emplois qu'elle assure au sein des activités de nombreuses régions rurales (voir tableau ci-après pour certains pays).

13 Mais cet état de fait, qui s'est progressivement imposé au fur et à mesure que l'agriculture perdait ses actifs excédentaires, s'est trouvé renforcé par le développement d'une fonction purement résidentielle. De plus en plus de familles viennent s'installer dans le milieu rural, tout en occupant un emploi en ville. Elles renforcent le contingent des ouvriers et employés autour des villes moyennes, celui des cadres et professions intermédiaires en périphérie des plus grandes métropoles. Les ouvriers constituent aujourd'hui le groupe le plus important dans la population rurale avec, pour la France, un taux supérieur à celui des villes. Dès lors, dans les pays industrialisés et urbanisés, les agriculteurs ne représentent guère plus de $10 \%$ des actifs ruraux et le taux ne dépasse $30 \%$ que dans un faible nombre de cas. Une autre caractéristique de la population rurale tient à la place qu'y occupent les retraités, conséquence du vieillissement des habitants et d'un apport de personnes qui viennent s'installer à la campagne pour leur retraite. En France, par exemple, leur part approche $40 \%$ des ménages. 
Quelques caractéristiques des régions rurales selon l'OCDE

\begin{tabular}{|c|c|c|c|c|c|}
\hline & $\begin{array}{c}\text { \% d'espace } \\
\text { rural }\end{array}$ & $\begin{array}{c}\text { densité } \\
\mathrm{hab} / \mathrm{km}^{2}\end{array}$ & $\begin{array}{l}\% \text { d'actifs } \\
\text { agricoles }\end{array}$ & $\begin{array}{l}\text { variation/an } \\
\text { population }{ }^{1}\end{array}$ & $\begin{array}{c}\% \text { de } \\
0-14 \text { ans }\end{array}$ \\
\hline Canada........... & 33 & 1 & 11 & +5.7 & 22.8 \\
\hline Royaume-Uni ........ & 15 & 67 & 10 & +6.7 & 18.9 \\
\hline Italie..... & 9 & 65 & 16 & +2.6 & - \\
\hline Norvège .... & 51 & 8 & 8 & +1.4 & - \\
\hline Portugal...$\ldots \ldots \ldots$ & 35 & 47 & 37 & - & 18.6 \\
\hline France $\ldots \ldots \ldots \ldots \ldots$ & 30 & 52 & 11 & +2.9 & 18.4 \\
\hline
\end{tabular}

1 Période 80/90.

* Régions essentiellement rurales selon l'OCDE.

La structure d'âge des ruraux est marquée par ce phénomène et souffre d'un incontestable vieillissement. Mais, contrairement à ce que beaucoup imaginent, le milieu rural n'est pas dépourvu de jeunes. En France toujours, le pourcentage des moins de quatorze ans est plus fort dans les communes rurales que dans les villes en raison de l'installation de jeunes ménages, c'est le cas de beaucoup d'autres pays d'Europe. Dans les pays moins développés, la natalité rurale est souvent très forte, si bien que partout la question de l'école demeure un point central du développement des zones rurales.

Aujourd'hui, la société rurale n'est plus une société agricole, elle est multiforme à la fois par les activités qu'y exercent les gens, par leur origine géographique, par les motivations qui les y ont amenés. Les nouveaux venus sont nombreux, ils introduisent des références et des modes de vie différents, et un brassage culturel qui ouvre la société sur l'extérieur. On peut ainsi affirmer que la nouvelle population rurale a de plus en plus choisi son milieu de vie.

\section{Nouvelles dynamiques d'évolution}

La principale tendance d'évolution du milieu rural a pendant longtemps été celle d'un lent déclin de la population et des activités. C'est encore le cas dans beaucoup de pays en retard de développement. Mais, dans les pays d'Europe et d'Amérique du Nord, l'analyse des tendances les plus récentes vient souvent contredire cette image, ou tout au moins la nuancer fortement ${ }^{3}$.

Sur le plan démographique d'abord, il est vrai que durant près d'un siècle un exode massif alimenté par les agriculteurs en surnombre a décimé la population de nombreux villages ruraux. Mais, depuis quinze/vingt ans, la tendance s'est inversée dans pratiquement tous les pays industrialisés : le solde des migrations est devenu positif dans une majorité de communes sous l'effet de l'arrivée de nouveaux ménages et de l'affaiblissement des départs. Les arrivants sont à la fois des jeunes ménages accédant à la propriété, des actifs accompagnant le redéploiement territorial de certaines activités, des retraités, des gens déçus des conditions de vie aujourd'hui offertes par les villes. Ces installations viennent plus que compenser le déficit naturel consécutif du vieillissement et de la baisse de la natalité, si bien que dans pratiquement tous les pays de l'OCDE la population des régions rurales est actuellement en croissance ${ }^{4}$. Cette croissance n'est cependant pas le fait de la totalité des zones rurales, elle est plus forte dans les espaces désignés sous le terme de 
périurbains, dans les zones d'industrialisation diffuse et dans celles de grand tourisme, mais elle est significative sur un vaste territoire ; en France, par exemple, plus de $60 \%$ des communes rurales ont enregistré des gains démographiques entre 1982 et 1990.

L'évolution des activités économiques est le second domaine d'importants changements. La modernisation des activités traditionnelles du milieu rural (agriculture, artisanat, anciennes industries...) s'est poursuivie avec les ajustements de main-d'œuvre que cela implique, c'est-à-dire d'importantes pertes d'emploi et la montée du chômage. Mais là n'est pas l'essentiel car de nouvelles dynamiques, plus positives, sont aujourd'hui à l'œuvre. On a assisté tout d'abord à un redéploiement territorial de certaines activités industrielles trop à l'étroit dans les tissus urbains de banlieue, qui ont cherché dans l'espace rural et les petites villes plus d'espace et de la main-d'œuvre à meilleur marché. S'il est en partie tari, ce mouvement a généré l'implantation de nombreuses unités petites et moyennes qui ont mieux résisté à la crise que les grands sites industriels. Cette attractivité a aussi joué pour des créateurs d'entreprises individuelles cherchant dans le milieu rural un cadre de vie autant qu'un cadre de travail. Des études réalisées dans plusieurs pays européens (France, Royaume-Uni, Italie) ont montré ce phénomène qui a apporté de nombreuses initiatives dans des créneaux innovants de l'industrie et du tertiaire, impliquant souvent les nouvelles technologies de communication. Ceci va de pair avec la mise en valeur - ou la redécouverte ! - de nouvelles ressources de l'espace rural. Les produits agricoles, de plus en plus conditionnés et transformés par des chaînes industrielles ont permis aux industries agroalimentaires de créer de nombreux emplois (c'est une des rares branches créatrices nettes d'emplois sur les vingt dernières années). Des savoir-faire locaux réhabilités et codifiés sont à l'origine du développement de petites entreprises, dispersées sur le territoire, jouant sur la qualité de leurs produits. La santé et la remise en forme sont une autre piste autour de laquelle se sont créés de nouveaux établissements. Le tourisme enfin a connu dans les régions rurales les plus propices un développement décisif pour toute l'économie locale. De façon directe (hôtellerie, accompagnement des activités de loisirs...) et plus encore indirecte (bâtiment, commerces et services), il a permis la création d'emplois nouveaux et de conforter les activités existantes par la pratique de la pluriactivité. C'est ainsi que certaines régions de montagne ou certaines îles qui semblaient, il y a cinquante ans, être vouées à l'abandon attirent de nouveaux habitants et connaissent aujourd'hui un essor incontestable.

Ces dynamiques de reconquête concernent aussi l'ensemble des infrastructures de l'espace rural. Le réseau routier a été considérablement amélioré, l'habitat réhabilité ou renouvelé par des constructions neuves aligne progressivement son niveau de confort sur celui des villes. Dans les pays les plus développés, le téléphone et les nouveaux moyens de communication ont désenclavé les villages. Les services de santé, l'école, la banque sont partout accessibles sous une forme ou sous une autre et vivre en milieu rural ne présente plus aujourd'hui les handicaps qui avaient, par le passé, provoqué l'exode de nombreux jeunes.

20 Tous ces mouvements ont des significations diverses, les uns étant soutenus par des dynamiques résidentielles, les autres par la mise en valeur des ressources du milieu ou l'apport de nouvelles entreprises; certains découlant de l'initiative locale, d'autres d'apports extérieurs ou de l'influence des villes proches. Ils n'affectent pas de la même manière tous les espaces : les plus enclavés et ceux demeurés les plus agricoles voient souvent la poursuite des tendances antérieures, mais, dans une majorité de cas, ces 
dynamiques de reconquête sont en germe ou à l'œuvre et expriment un nouveau rapport de la société à son territoire.

21 L'espace rural assume maintenant de nouvelles fonctions qui vont bien au-delà de la production agricole et forestière : il est le cadre résidentiel de ménages pratiquant un mode de vie intégrant une forte mobilité, le lieu de développement d'initiatives économiques innovantes, l'espace de récréation d'une large partie de la population ; il est aussi de plus en plus un lieu de reproduction des grands équilibres naturels et de conservation d'un patrimoine paysager et de savoir-faire traditionnels.

Dans ce nouveau contexte rural, l'organisation et la modernisation du service public, et en particulier de l'école, sont des enjeux majeurs. L'école doit en effet non seulement remplir sa mission d'offrir une égalité de chances à tous, mais contribuer à l'intégration du monde rural à la société future. C'est un champ considérable pour l'innovation, en termes de modalités d'adaptation aux conditions de la faible densité comme en termes de contenu pédagogique.

\section{NOTES}

1. Créer des indicateurs ruraux pour étayer la politique territoriale, Paris, OCDE, 1994, 97 p. Le seuil retenu pour qualifier les collectivités rurales est de $150 \mathrm{hab} / \mathrm{km}^{2}$, sauf au Japon où il est de 500 .

2. J.-C. Bontron, «Le concept de ruralité à l'épreuve du changement social », in: Territoires ruraux et développement, Quel rôle pour la recherche?, Cemagref Éditions/Association Descartes, 1994, p. $162-166$.

3. J.-C. Bontron, « L’Europe rurale est-elle menacée de désertification ? » in : L'événement européen, Initiatives et débats, « Europe : espace ou territoire ? », février 1993/21, p. 157-167.

4. Voir tableau.

\section{RÉSUMÉS}

Les tendances générales d'évolution dans les pays de l'OCDE bouleversent les représentations traditionnelles qui associaient, du point de vue économique, social et démographique, le milieu rural au secteur agricole. Dans des situations nationales certes fort diversifiées, ces transformations récentes doivent être prises en compte dans la problématique de la politique scolaire. 
INDEX

Index géographique : Pays de l'OCDE

Mots-clés : développement rural, école rurale, milieu rural, politique éducative

\section{AUTEUR}

JEAN-CLAUDE BONTRON

Directeur de la Société d'études géographiques, économiques et sociologiques appliquées (SEGESA), Paris, France. 